\title{
Lack of hepatic stimulator substance expression promotes hepatocellular carcinoma metastasis partly through ERK-activated epithelial-mesenchymal transition
}

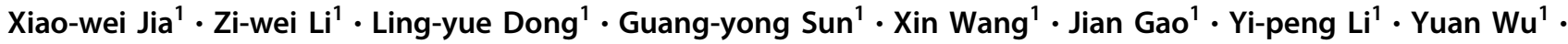 \\ Wei $\mathrm{An}^{1}$
}

Received: 11 August 2017 / Revised: 18 January 2018 / Accepted: 30 January 2018 / Published online: 1 March 2018

(c) United States \& Canadian Academy of Pathology 2018

\begin{abstract}
Hepatocellular carcinoma (HCC) is one of the most lethal malignancies due to its high frequency of metastasis via the epithelial-mesenchymal transition (EMT) pathway. Hepatic stimulator substance (HSS) can protect hepatocytes from injury and promote liver growth. Recent studies indicated that HSS expression is increased in HCC tissues; however, whether HSS expression is potentially associated with HCC metastasis, particularly through the EMT pathway, remains largely unknown. In this study, the relationship between HSS expression and HCC metastasis was investigated in clinical samples of HCC. Meanwhile, the regulation of HCC metastasis and EMT progression by HSS were also analyzed in both in vitro and in vivo models. The results showed that the expression of $23 \mathrm{kDa}$ HSS was significantly decreased among HCC tissues with angioinvasion. A decrease in HSS predicted poor prognosis with a lower survival rate. Furthermore, the growth of xenograft tumors after inoculating MHCC97H-HSS-shRNA (HCC) cells into nude mice was notably accelerated compared to those inoculated with HSS-expressing cells. Further analysis revealed that knockdown of HSS expression in both MHCC97H and HepG2 cells could enhance the migration of these HCC cells. Concurrently, interference of HSS expression by shRNA promoted conversion of morphologically epithelial-like HCC cells into mesenchymal-like cells, together with downregulations of epithelial markers (such as E-cadherin and zonula occludens-1) and upregulation of mesenchymallike makers (such as $\alpha$-SMA, $\beta$-catenin, and fibronectin). Furthermore, it was demonstrated that, as well as promoting EMT, $H S S$-shRNA induced the phosphorylation of extracellular signal-regulated kinase (ERK) and elevated the expression of the EMT-related transcription factor Snail. Specific inhibition of HSS-shRNA-induced ERK phosphorylation by PD98059 attenuated HCC cell migration in a dose-dependent manner. In conclusion, we demonstrated that downregulation of HSS expression contributes to HCC metastasis partially through the ERK-activated EMT pathway.
\end{abstract}

\section{Introduction}

Hepatocellular carcinoma (HCC) is one of the most prevalent malignant cancers worldwide [1]. The development and progression of $\mathrm{HCC}$ in humans are complicated

These authors contributed equally: Xiao-wei Jia and Zi-wei Li.

Wei An

anwei@ccmu.edu.cn

1 Department of Cell Biology and the Municipal Laboratory of Liver Protection and Regulation of Regeneration, Capital Medical University, 10 You An Men Wai Xi Tou Tiao, 100069 Beijing, China processes involving multiple genes and steps [2]. Currently, surgical resection of the tumor is the first and most effective choice for earlier-stage HCC; however, the prognosis remains poor. The major reason for this poor prognosis is that most symptomatic HCC patients, when diagnosed, are actually at an advanced stage. Irrespective of surgical resection, long-term outcomes of HCC are still unsatisfactory because of remote metastases and frequent recurrence after surgery. It is believed that, during HCC progression, the epithelial-mesenchymal transition (EMT) has an essential role in the early steps of metastasis when cells lose cell-cell contacts due to loss of E-cadherin, and consequently acquire increased motility to spread into surrounding or distal tissues. It is well known that the EMT is a crucial mechanism underlying the "escape" of epithelial-originating malignant hepatocytes from basement 
membrane barriers and stroma into blood or lymphatic vessels. The EMT also has an important role in HCC migration. It is reported that various cytokines or growth factors such as tumor growth factor- $\beta$ (TGF- $\beta$ ) and hepatocyte growth factor (HGF) can inhibit the expression of E-cadherin and increase the expression of $\beta$-catenin, inducing EMT occurrence in the cancers and enhancing cancer cell mobility and metastasis [3, 4]. Therefore, elucidation of the molecular mechanisms of EMT occurring in HCC has become one of the priorities for combating this threatening disease.

Hepatic stimulator substance (HSS) is a hepatotrophic protein that was initially identified in crude extract of liver homogenate in weanling rats [5]. As a unique liveroriginated growth factor, HSS was able to exclusively promote DNA synthesis and proliferation of hepatocytes $[6,7]$. However, in primary hepatocytes, HSS was proved to stimulate cell growth only when used in combination with other mitogens such as epidermal growth factor or hepatocyte growth factor [8], i.e., HSS itself could not exert a mitogenic effect in resting-stage hepatocytes. Because of this property, HSS was also known as augmenter of liver regeneration (ALR) [9]. A few years later, the $A L R$ gene was cloned, and its protein was successfully identified [10, 11]. In vertebrates, the $A L R$ gene is highly homologous to the yeast erv 1 (essential for respiration and viability 1) gene, which is required for mitochondrial metabolism [12]. In mammals, the HSS protein localizes in different organelles, but the mitochondrial intermembrane space (MIMS) is the major site [13, 14]. HSS possesses a conserved CXXC motif and a noncovalent flavin adenine dinucleotide (FAD) adjacent to CXXC $[15,16]$, which is crucial for HSS function, either as an FAD-dependent sulfhydryl oxidase responsible for mitochondrial protein import or as a cytochrome c reductase which helps transfer protons to MIMS $[17,18]$. Human HSS protein consists of two splice variants, a large isoform of $23 \mathrm{kDa}$ and a shorter isoform of 15 $\mathrm{kDa}$ [19]. It is now recognized that HSS is a survival factor for the liver [20], as depletion of HSS expression in hepatocytes not only increases the susceptibility of cells exposed to external insults [21], but also impairs liver regeneration after partial hepatectomy (PH) [22, 23]. In relation to HSS protection of hepatocytes, HSS protects mitochondria from oxidative damage [24, 25], alleviates endoplasmic reticulum (ER) stress [26], and inhibits apoptosis [27].

Although a large number of studies have underlined the role of HSS in regulation of liver protection and regeneration, and even its interplay with some liver diseases including steatosis [28], fibrosis [29], cirrhosis [29, 30], and acute liver failure [31], only a few investigations have addressed the relationship of HSS to HCC pathogenesis and HCC metastasis as well. Recently, Dayoub et al. [32] showed that the expression of one isoform of HSS (15 kDa) was inversely correlated with HCC metastasis, and was believed to be associated with enhanced progression of EMT. However, the study by Dayoub et al. still lacks direct information about the association of HSS with EMT in human HCC tissues and the molecular pathway through which HCC promotes EMT. More recently, Yu et al. [33] reported that the 23-kDa HSS isoform was highly expressed in HCC, but unfortunately, no further supporting data were provided in that study to interpret the molecular link of HCC to HSS expression.

In this study, we found that the expression of 23-kDa HSS was remarkably decreased in HCC tissues with angioinvasion and that decreased HSS expression predicted poor prognosis, reflected by survival rate. The knockdown of $H S S$ expression enhanced HCC cell metastasis in both in vitro and in vivo models. Meanwhile, the EMT markers were clearly manifested along with HCC metastasis. HSS-short hairpin RNA (shRNA) induced phosphorylation of extracellular signal-regulated kinase (ERK) and increased Snail expression in parallel with EMT progression in HCC cells, whereas the inhibition of ERK phosphorylation not only restricted EMT progression, but also suppressed HCC metastasis. In conclusion, our results suggest that the expression of HSS is closely associated with HCC metastasis and therefore might affect the outcome in HCC patients.

\section{Materials and methods}

\section{Human HCC tissue samples}

Liver tissues were obtained from 44 patients with primary HCC who had undergone hepatic resection between July 2010 and January 2012 at Beijing You'an Hospital (Beijing, China). The clinical characteristics of patients with HCC are shown in Table 1. The diagnosis of HCC was based on histological examination. Liver tissues were snap frozen immediately after surgical resection and stored at $-80^{\circ} \mathrm{C}$ for protein and RNA extraction. The experimental procedures were approved by the Ethics Committee of Capital Medical University, and written informed consent was obtained from each patient.

\section{RNA extraction and quantitative real-time PCR}

Total RNA extraction, first-strand cDNA synthesis, and quantitative real-time PCR (qRT-PCR) were performed as described previously [34]. qRT-PCR was performed to assess $H S S$ gene expression. The primers used to amplify HSS were as follows: sense, 5'-TGAAGCCCAAATGAAACGC-3'; antisense, 5'-CACAGAGGGATGGAACAAGC-3'. Amplicon expression in each sample was normalized to $\beta$-actin. Primers used to amplify $\beta$-actin were as follows: sense, 
Table 1 The associations of HSS expression with clinicopathological characteristics in $\mathrm{HCC}$ patients

\begin{tabular}{|c|c|c|c|}
\hline Feature & $\begin{array}{l}\text { Low expression of } \\
H S S(n=19)\end{array}$ & $\begin{array}{l}\text { High expression of } \\
H S S(n=25)\end{array}$ & $P$ value \\
\hline \multicolumn{4}{|l|}{ Gender } \\
\hline Male & 18 & 19 & \multirow[t]{2}{*}{0.205} \\
\hline Female & 1 & 6 & \\
\hline \multicolumn{4}{|l|}{ Age (years) } \\
\hline$\leq 50$ & 11 & 12 & \multirow[t]{2}{*}{0.515} \\
\hline$>50$ & 8 & 13 & \\
\hline \multicolumn{4}{|l|}{$\mathrm{HBsAg}$} \\
\hline Negative & 0 & 5 & \multirow[t]{2}{*}{0.112} \\
\hline Positive & 19 & 20 & \\
\hline \multicolumn{4}{|l|}{ AFP (ng/ml) } \\
\hline$\leq 20$ & 6 & 15 & \multirow[t]{2}{*}{0.062} \\
\hline$>20$ & 13 & 10 & \\
\hline \multicolumn{4}{|l|}{ Cirrhosis } \\
\hline No & 1 & 2 & \multirow[t]{2}{*}{1.001} \\
\hline Yes & 18 & 23 & \\
\hline \multicolumn{4}{|c|}{ Tumor size $(\mathrm{cm})$} \\
\hline$\leq 5$ & 12 & 12 & \multirow[t]{2}{*}{0.317} \\
\hline$>5$ & 7 & 13 & \\
\hline \multicolumn{4}{|c|}{ Tumor number } \\
\hline Single & 9 & 19 & \multirow[t]{2}{*}{0.051} \\
\hline Multiple & 10 & 6 & \\
\hline \multicolumn{4}{|c|}{ Vascular invasion } \\
\hline No & 7 & 23 & \multirow[t]{2}{*}{$0.001 *$} \\
\hline Yes & 12 & 2 & \\
\hline \multicolumn{4}{|c|}{$\begin{array}{l}A F P \alpha \text {-fetoprotein, } H B s A g \text { hepatitis } \mathrm{B} \text { surface antigen, } H C C \\
\text { hepatocellular carcinoma }\end{array}$} \\
\hline \multicolumn{4}{|c|}{ *Significance was evaluated using Pearson's $\chi^{2}$ test or Fisher's exact test } \\
\hline \multicolumn{4}{|c|}{$\begin{array}{l}\text { 5'-ACCCACACTGTGCCCATCTA-3'; antisense, 5'- } \\
\text { GCCACAGGATTCCATACCCA-3'. After normalization, } \\
\text { gene expression was quantified using the } 2^{-\Delta \Delta C t} \text { method } \\
\text { [35]. }\end{array}$} \\
\hline
\end{tabular}

\section{Cell culture}

HepG2 cells were obtained from the American Type Culture Collection (ATCC, Manassas, VA, USA) and MHCC97H cells were obtained from Guangzhou Jennio Biotech (Guangzhou, China). All cells were cultured in Dulbecco's modified Eagle medium (DMEM) with $10 \%$ fetal bovine serum (FBS), $100 \mathrm{U} / \mathrm{mL}$ penicillin and $100 \mu \mathrm{g} / \mathrm{mL}$ streptomycin.

\section{Establishing and culturing HSS-expressing or HSS- knockdown cell lines}

Both HepG2 and MHCC97H cells were stably transfected with the HSS- or HSS-shRNA-containing plasmids. To establish stable $H S S$-knockdown cells, a shRNA plasmid targeting the HSS transcript was obtained from GenePharma (Shanghai, China) and the sequence was 5'-GAA GCGGGACACCAAGTTTAG-3'. The cells were transfected with $5 \mu \mathrm{g}$ of either $H S S$-shRNA or scramble vector with Lipofectamine ${ }^{\mathrm{TM}} 2000$ (Thermo Fisher Scientific, Waltham, MA, USA) according to the manufacturer's recommendations. At $24 \mathrm{~h}$ post-transfection, the cells were selected using Geneticin (G-418, Solarbio, Beijing, China) $(800 \mu \mathrm{g} / \mathrm{mL})$ for 14 days. Stable $H S S$-expressing (HSS-Tx) or HSS-knockdown (HSS-shRNA) clones were confirmed by qRT-PCR and western blot analysis.

\section{Western blotting}

The cells, after completion of different treatments, were harvested and lysed, and total protein was extracted. After determination of concentration, proteins were separated using SDS-polyacrylamide gel electrophoresis (PAGE) and transferred to a nitrocellulose membrane. Primary antibodies against specific markers were used, which included HSS (diluted 1:1000; Proteintech, Chicago, IL, USA), Zonula occludens-1 (ZO-1, diluted 1:1000; Abcam, Cambridge, UK), E-cadherin (E-cad, diluted 1:1000; Cell Signaling Technology, Beverly, MA, USA), $\beta$-catenin (diluted 1:1000; Cell Signaling Technology, Beverly, MA, USA), $\alpha$-smooth muscle actin ( $\alpha$-SMA, diluted 1:1000; Sigma-Aldrich, St. Louis, MO, USA), fibronectin (diluted 1:1000; Abcam, Cambridge, UK), or glyceraldehyde 3-phosphate dehydrogenase (GAPDH, diluted 1:10,000; KangChen, Shanghai, China). The relative density of the protein bands was quantitatively determined using Image $\mathrm{J}$ software (National Institutes of Health, Bethesda, MD, USA).

\section{Migration assay}

HepG2 cells were resuspended in FBS-free DMEM and seeded into Boyden chambers with $8-\mu \mathrm{m}$ filter pores inserted into 24-well plates. FBS (1\%) served as a chemoattractant in the lower chamber, and FBS-free DMEM was added to the upper chamber. After $24 \mathrm{~h}$, the cells were fixed and stained with 4',6-diamidino-2-phenylindole (DAPI) or hematoxylin, and the migrated cells were counted. Migration was quantified by analyzing at least six random fields per triplicate filter for each independent experiment using an inverted fluorescence microscope.

\section{In vivo experiments}

Four-week-old male athymic BALB/c mice were purchased from the Beijing Vital River Laboratory Animal Technology Co. Ltd. (Beijing, China) and maintained under 


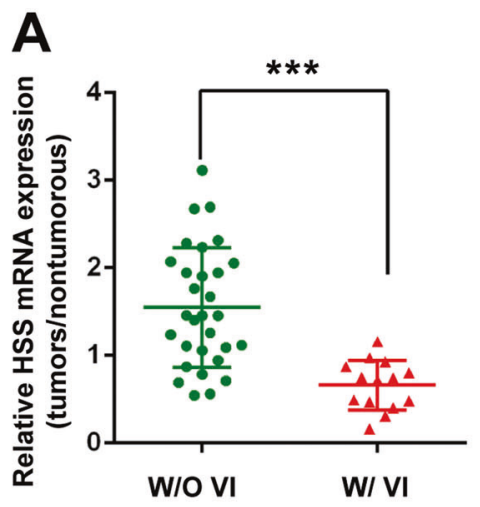

WIO VI: Without vascular invasion WI VI: With vascular invasion

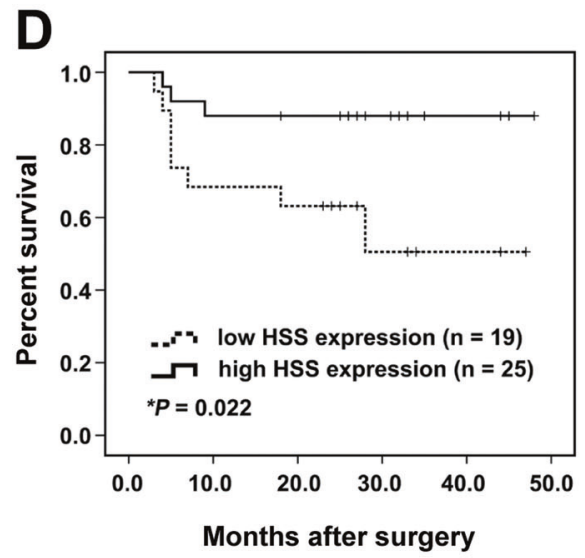

Months after surgery

\section{B}

Without vascular invasion cases(HSS/DAPI)
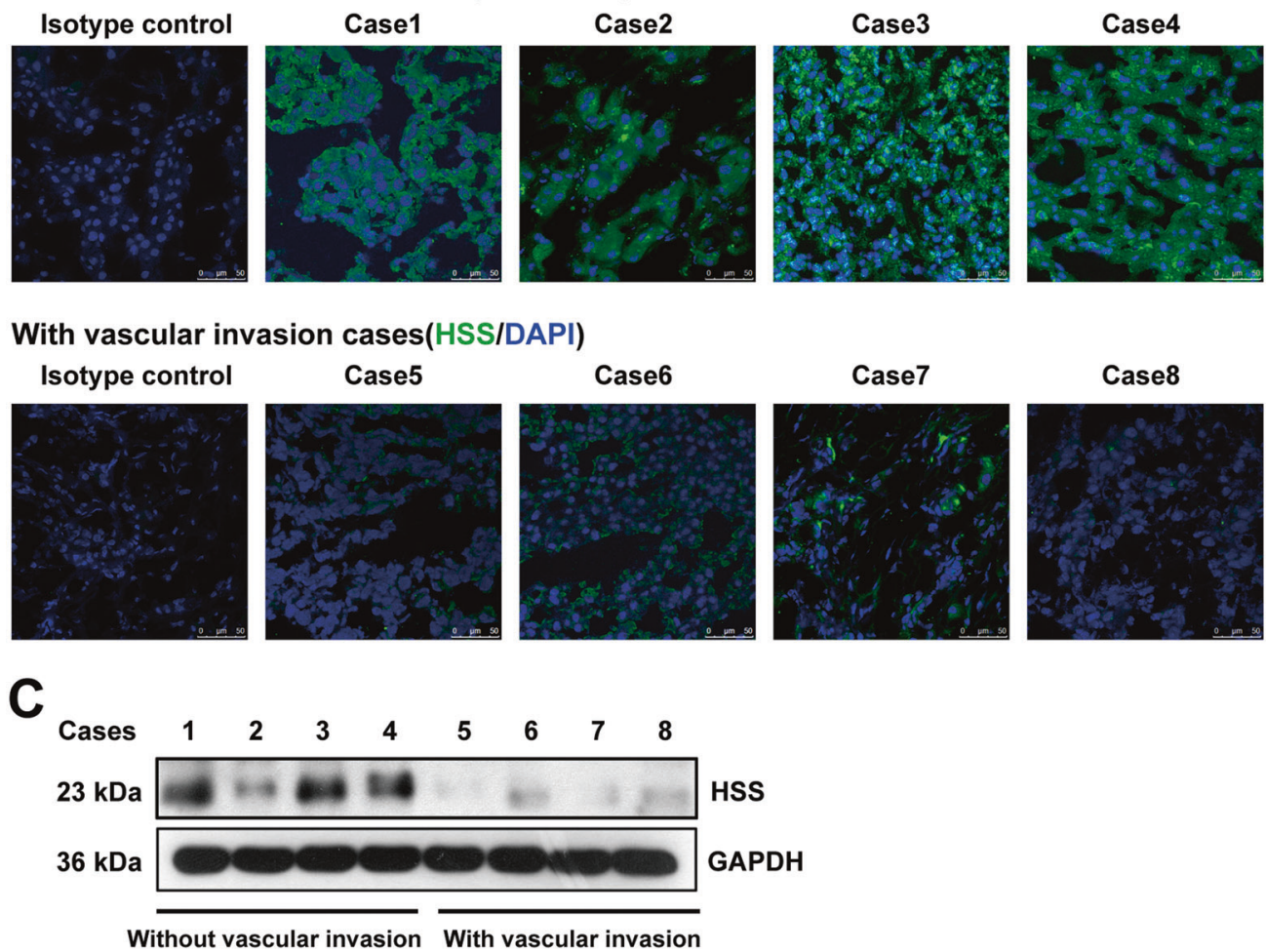

Fig. 1 HSS expression in HCC tissue samples. a Samples from 44 cases of patients with HCC were collected and relative HSS mRNA expression was tested by qRT-PCR. qRT-PCR measurement of HSS transcript was normalized to $\beta$-actin transcript in 44 cases. Data are expressed as mean $\pm \mathrm{SD}$, $* * * P<0.001$. HSS mRNA expression is significantly decreased in HCC tissues with vascular invasion as compared with those of non-vascular invasion, which were corrected with adjacent non-tumor tissues. b HCC patients with HSS high

controlled conditions with a $12 \mathrm{~h}$ light-dark cycle. All protocols related to animal management were performed in accordance with the guidelines of the Ethics Committee of the Capital Medical University.

MHCC $97 \mathrm{H}$ cells were used to examine the role of HSS transfection in the growth and metastasis of xenograft expression (solid line) had better survival rate comparing with patients with low expression $(P=0.022)$. $\mathbf{c}$ HSS expression was displayed in eight cases of HCC patients by immunofluorescence. HSS high expression was seen in the samples without vascular invasion. In contrast, HSS low expression was shown in the HCC tissue with vascular invasion. Isotype control was used as negative control. d Western blot confirmed that $23 \mathrm{kDa}$ HSS expression was downregulated in the cases with vascular invasion.

tumors. An aliquot of $1 \times 10^{6}$ MHCC $97 \mathrm{H}-\mathrm{HSS}$-shRNA cells was suspended in $200 \mu \mathrm{L}$ PBS (phosphate-buffered solution) and injected subcutaneously into the right armpits of mice to evaluate whether xenograft tumors could be generated. Meanwhile, MHCC $97 \mathrm{H}-H S S$-Tx cells were injected into the left armpits. Tumor growth 
A ${ }_{\text {MHCC97H-HSS-Tx }}$

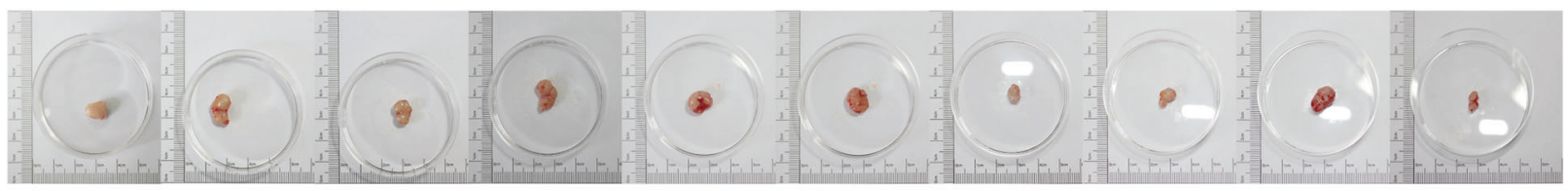

MHCC97H-HSS-shRNA

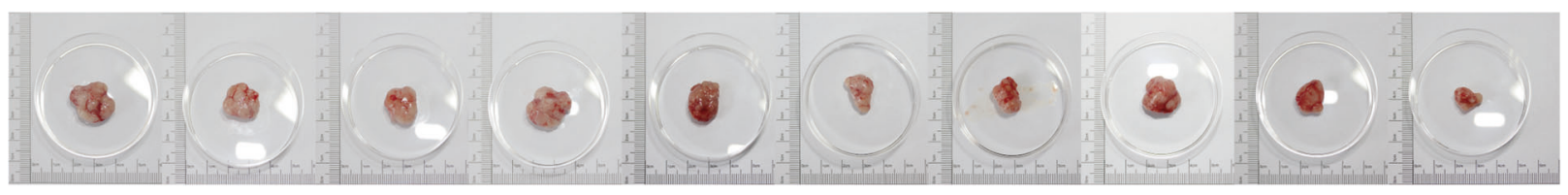

B

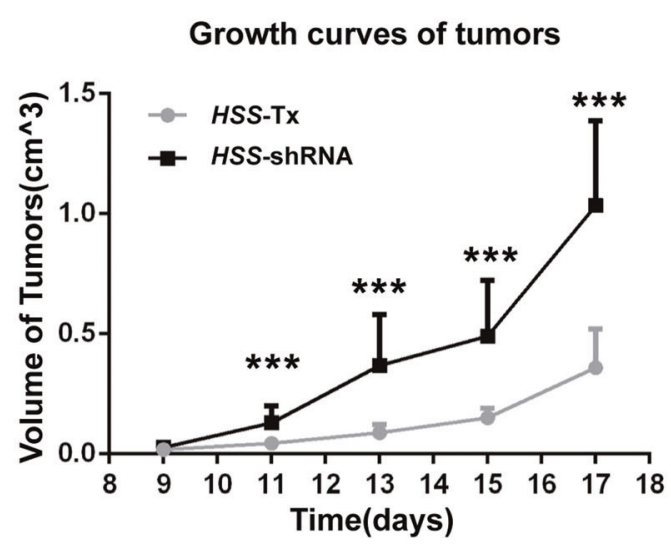

D
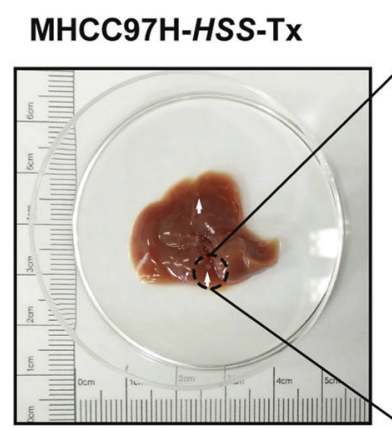

MHCC97H-HSS-shRNA
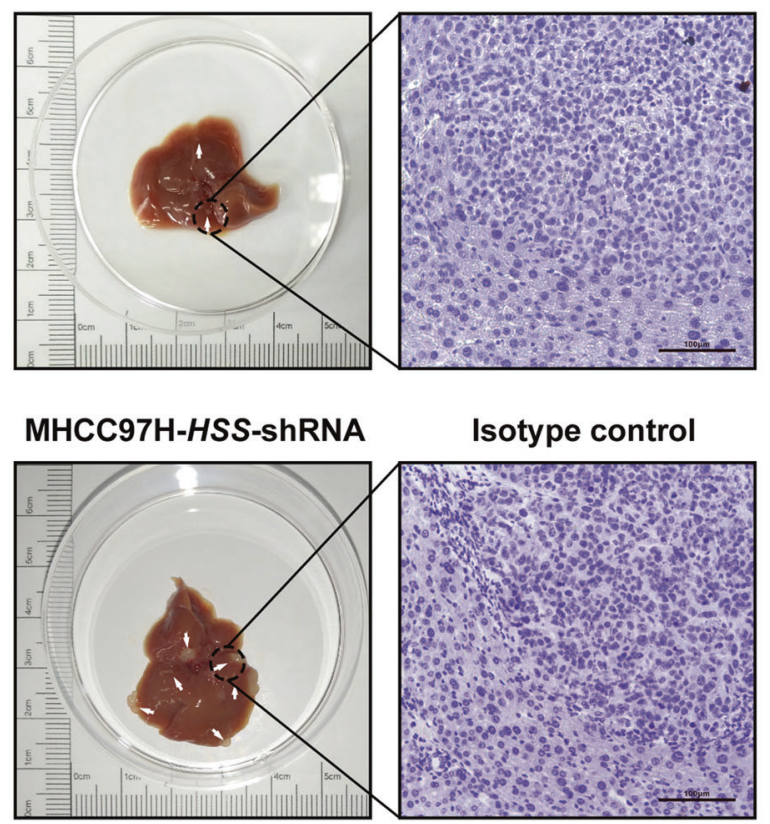

C Weight of tumors

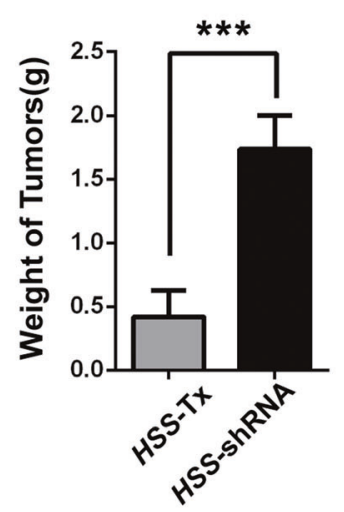

HSS staining
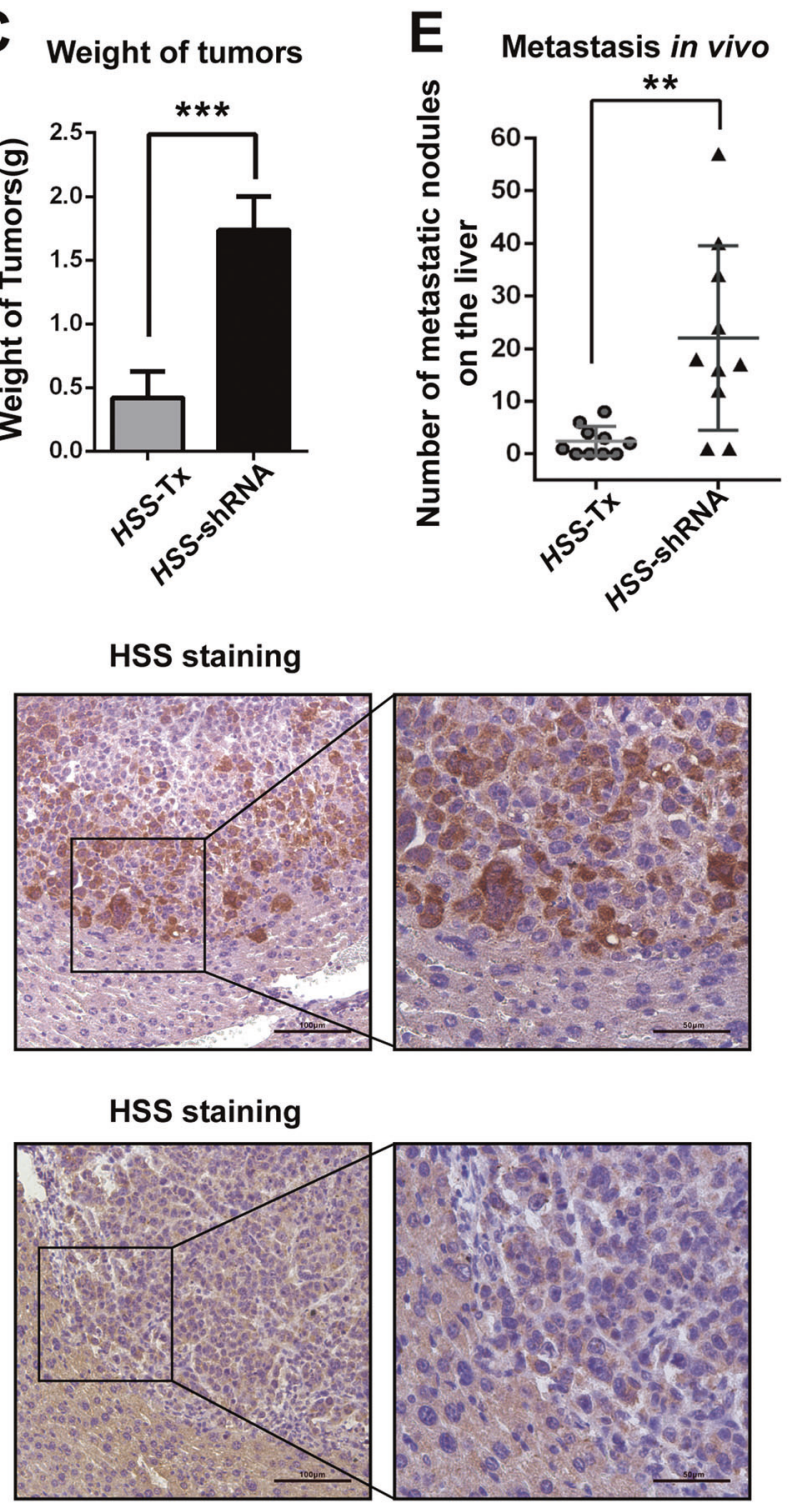

was examined daily for at least 5 weeks. The length and width of tumors were measured using calipers every 3 days once they could be palpated, and tumor volume was calculated using the formula, tumor volume $=$ length $\times$ width $^{2} / 2$. Tumor weights were determined at the end of the experiment. 
Fig. $2 H S S$-shRNA enhanced HCC cell proliferation and metastasis in vivo. a Xenograft tumor sizes generated by subcutaneously inoculating $1 \times 10^{6}$ MHCC97H-HSS-Tx cells (shorten as HSS-Tx) and MHCC 97H-HSS-shRNA cells (shorten as HSS-shRNA) to the right and left armpits of nude mice $(n=10)$. b The growth of xenograft tumor was significantly reduced in the tumors caused by $\mathrm{MHCC} 97 \mathrm{H}-$ $H S S$-Tx than those caused by MHCC97H-HSS-shRNA $(n=10)$. c The average weight of MHCC $97 \mathrm{H}-\mathrm{HSS}$-shRNA tumors was much higher than MHCC $97 \mathrm{H}-H S S-T x$ tumors $(n=10)$. d The statistics of spleento-liver metastatic HCC nodules caused by inoculation of MHCC97H$H S S$-shRNA or MHCC97H-HSS-Tx cells. The IHC assay was conducted to detect the HSS expression in tumors of MHCC97H cells and isotype control was used as negative control. e The numbers of spleento-liver metastatic HCC nodules generated by MHCC97H-HSSshRNA were abundant as compared with those caused by $\mathrm{MHCC} 97 \mathrm{H}$ HSS-Tx. $* P<0.05, * * P<0.01$, and $* * * P<0.001$

In addition to xenograft tumor in situ, MHCC97H-HSSTx or MHCC97H-HSS-shRNA cells $\left(1 \times 10^{6}\right)$ were injected into the spleens of athymic BALB/c mice to investigate the metastatic ability of the tumor to the liver. After 6 weeks, the mice were killed, and the recipient livers were dissected, fixed in $4 \%$ phosphate-buffered paraformaldehyde overnight at $4{ }^{\circ} \mathrm{C}$ and prepared for histological analysis.

\section{Data analysis}

The results of multiple observations are presented as the mean \pm SD of at least three independent experiments. The data were analyzed using the statistical software SPSS 17.0 (IBM, Armonk, NY, USA), and differences between various groups were analyzed with one-way analysis of variance (ANOVA). $* P<0.05$ was considered statistically significant.

\section{Results}

\section{HSS expression was inversely correlated with HCC metastasis}

The correlations between $H S S$ expression and pathological features were analyzed in the liver samples of 44 patients with HCC. According to pathological reports, HCC tissue samples were divided into two groups, with or without angioinvasion (vascular invasion, VI). The results indicated that HSS mRNA expression was significantly decreased in HCC tissues with VI as confirmed by pathological diagnosis (Fig. 1a). The decrease in HSS expression in the VI-HCC tissues was confirmed by in situ immunofluorescence assay (Fig. 1b) and western blot analysis (Fig. 1c). These results suggest that HSS expression might be inversely correlated to HCC metastasis. Conversely, based on the HSS mRNA expression levels in HCC tissues, the patients were also divided into two groups, the HSS high-expression group (whose fold change in relative expression was higher than the mean) and the low-expression group (whose fold change in relative expression was lower than the mean) (Table 1). Among $25 \mathrm{HCC}$ patients with high HSS expression, only two cases $(2 / 25)$ were confirmed with VI, whereas in contrast, among patients with low HSS expression, 12 out of 19 cases presented with VI $(P=0.001)$. Importantly, after a 4-year follow-up, patients with high HSS expression had better survival rates $(88.0 \%)$ compared to those in patients with low HSS expression (57.9\%; $P=0.022$, Mantel-Cox test, Fig. 1d). Therefore, the expression of HSS in HCC tissue could be taken as a prognostic marker to predict the disease outcome in patients with HCC.

\section{HSS knockdown promoted cells growth and metastasis in vivo}

As shown in Fig. 2a and b, the growth of xenograft tumors formed by MHCC97H-HSS-shRNA cells was notably faster compared with those formed by MHCC97H-HSS-Tx. As shown in Fig. 2b and c, the knockdown of HSS expression by shRNA accelerated tumor growth and increased tumor weights. To analyze the ability of xenograft tumors to invade proximal tissues, MHCC97H-HSS-Tx or MHCC97H-HSS-shRNA cells were inoculated into the spleens of mice and the formation of metastatic tumors in the livers of the same mice was investigated. As shown in Fig. $2 \mathrm{~d}$ and e, the numbers of metastatic nodules resulting from MHCC97H-HSS-shRNA cells were significantly higher than those caused by MHCC97H-HSS-Tx cell inoculation. These in vivo results further support the hypothesis that the knockdown of HSS expression accelerates HCC tumor growth and promotes HCC metastasis.

\section{HSS knockdown enhanced cell migration in vitro}

To confirm the roles of HSS in HCC tumor invasion, the transwell assay was performed. The results shown in Fig. 3 suggest that $H S S$-shRNA accelerated migrations in the two HCC cell lines (MHCC97H and HepG2) tested, whereas $H S S$ transfection slowed down HCC cell migration. These in vitro data confirm the initial results showing that a decrease in HSS expression in HCC cells enhances their migratory abilities.

\section{Regulation of HSS expression affects the EMT}

The foregoing results clearly indicated that HSS downregulation was apparently involved in HCC metastasis. EMT is suspected to be one of the key mechanisms that result in tumor metastasis [36, 37]. To elucidate the potential regulatory effect of $H S S$ on HCC metastasis with respect to EMT progression, several EMT biomarkers were 


\section{HepG2 cells}

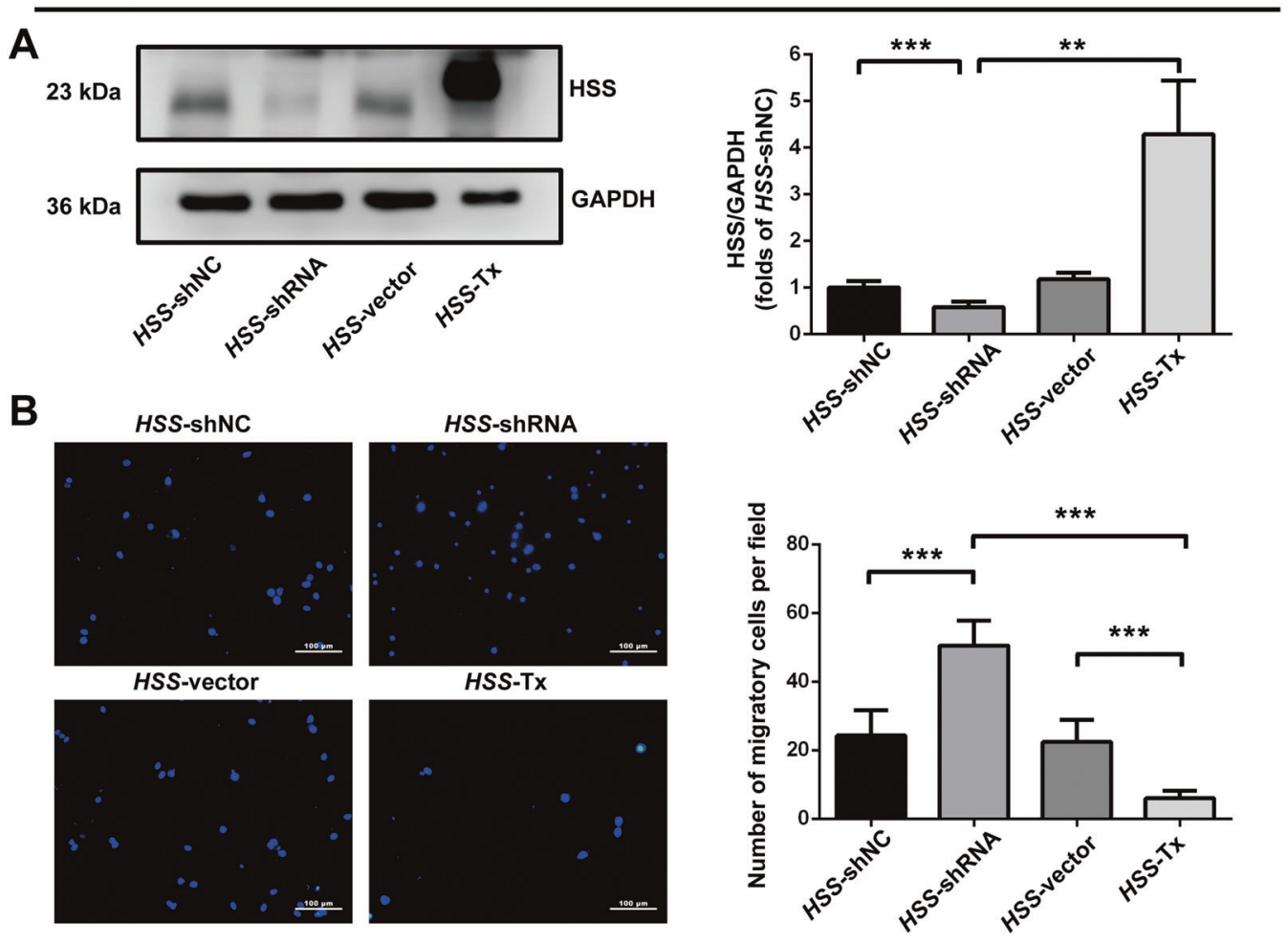

MHCC97H cells
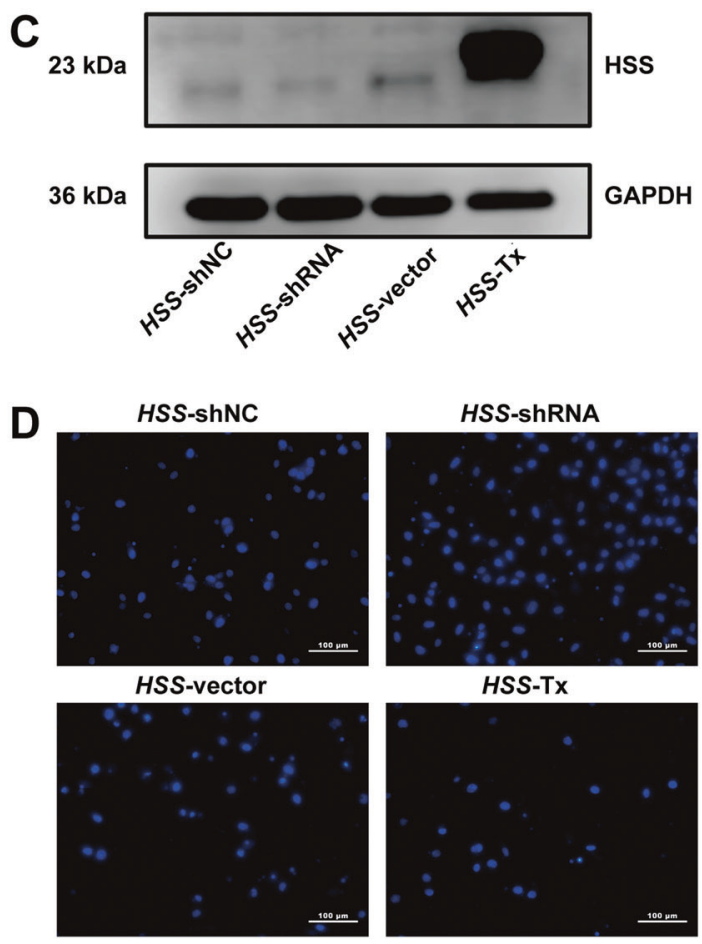
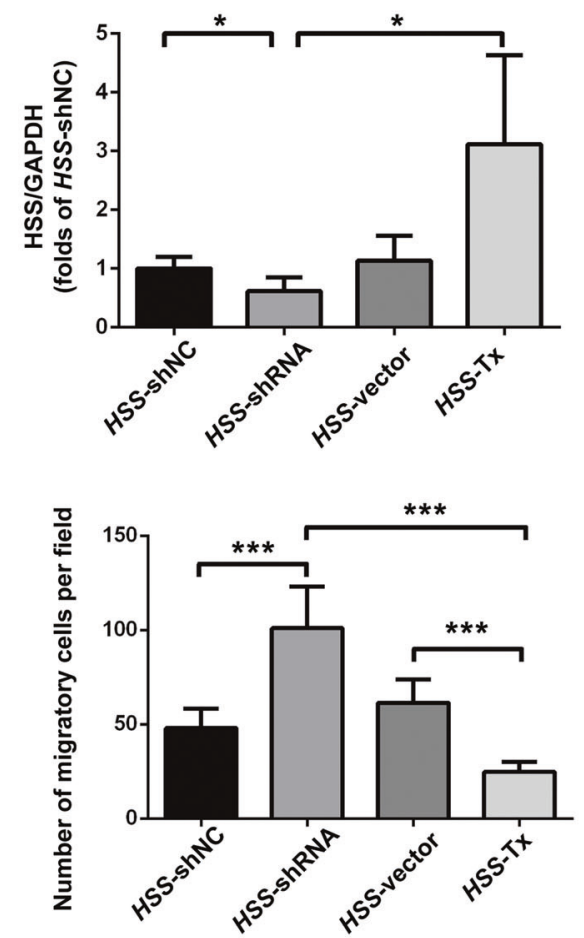

Fig. 3 Migratory capacities displayed by HSS-Tx or HSS-shRNA HCC cells. a, b Transwell assay and histograms in HepG2 cells. c, d Transwell assay and histograms in MHCC97H cells. The values are

expressed as the means $\pm \mathrm{SD}$ of three independent experiments. $* P<0.05, * * P<0.01$, and $* * * P<0.001$. 


\section{HepG2 cells}

\section{A}

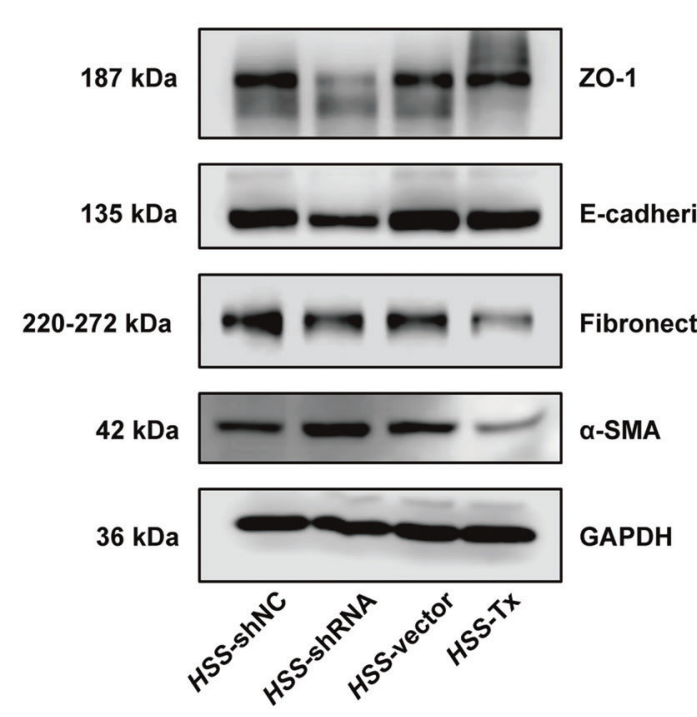

\section{B}
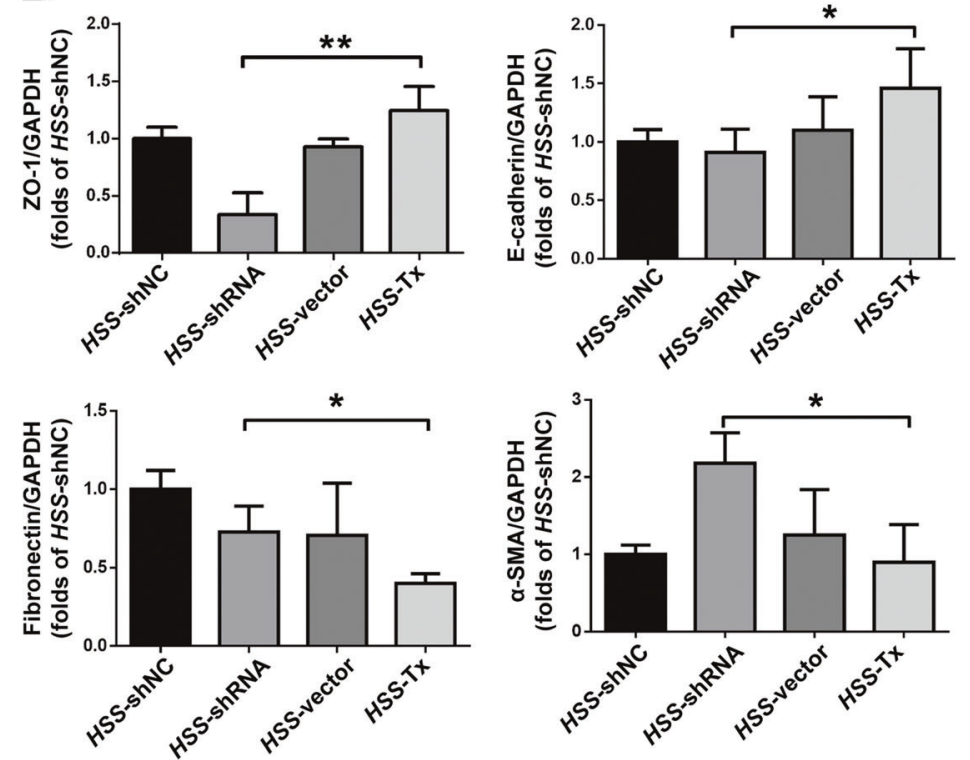

\section{Tumors of MHCC97H cells}
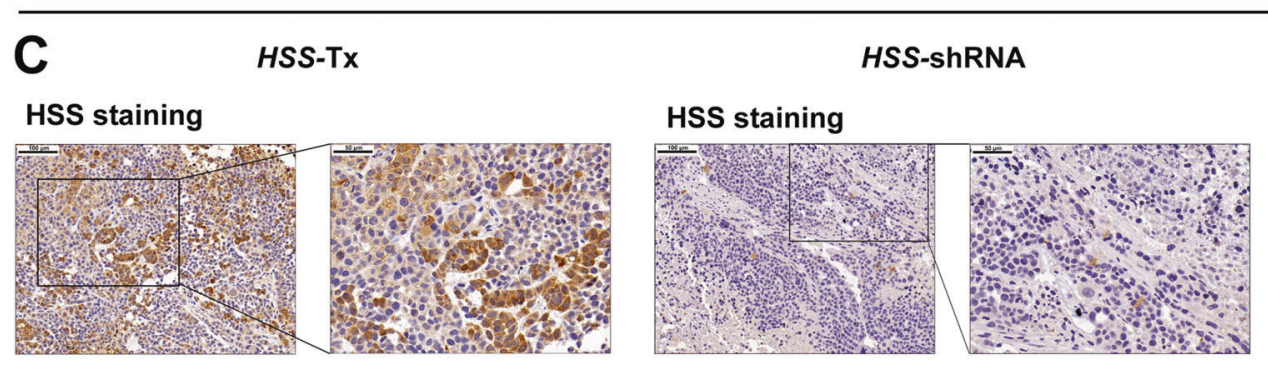

D
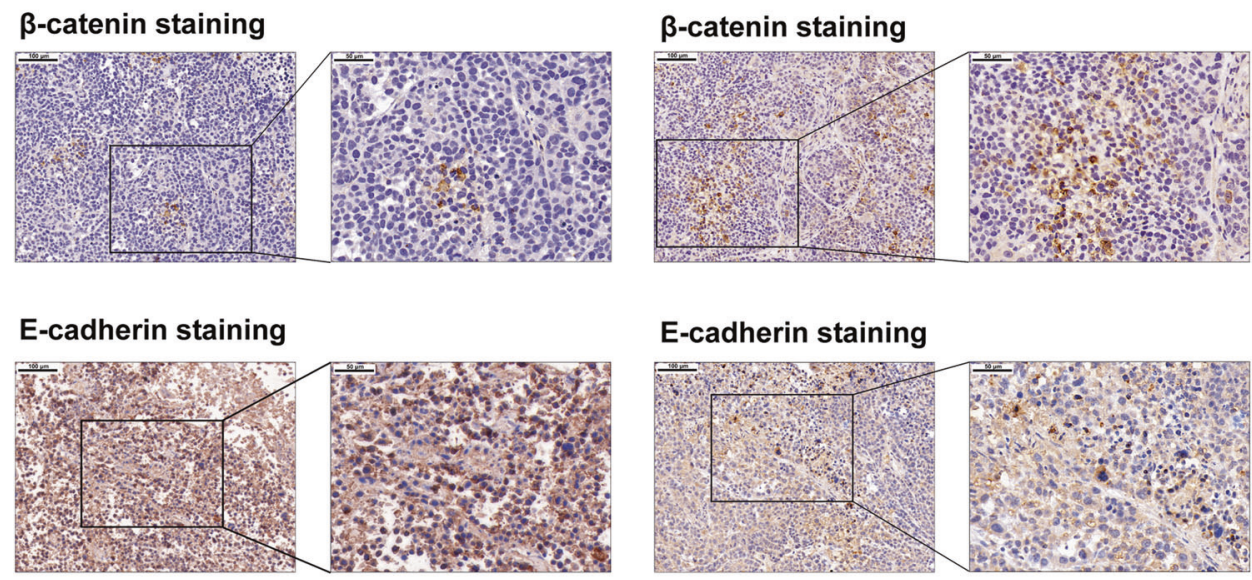

\section{E-cadherin staining}
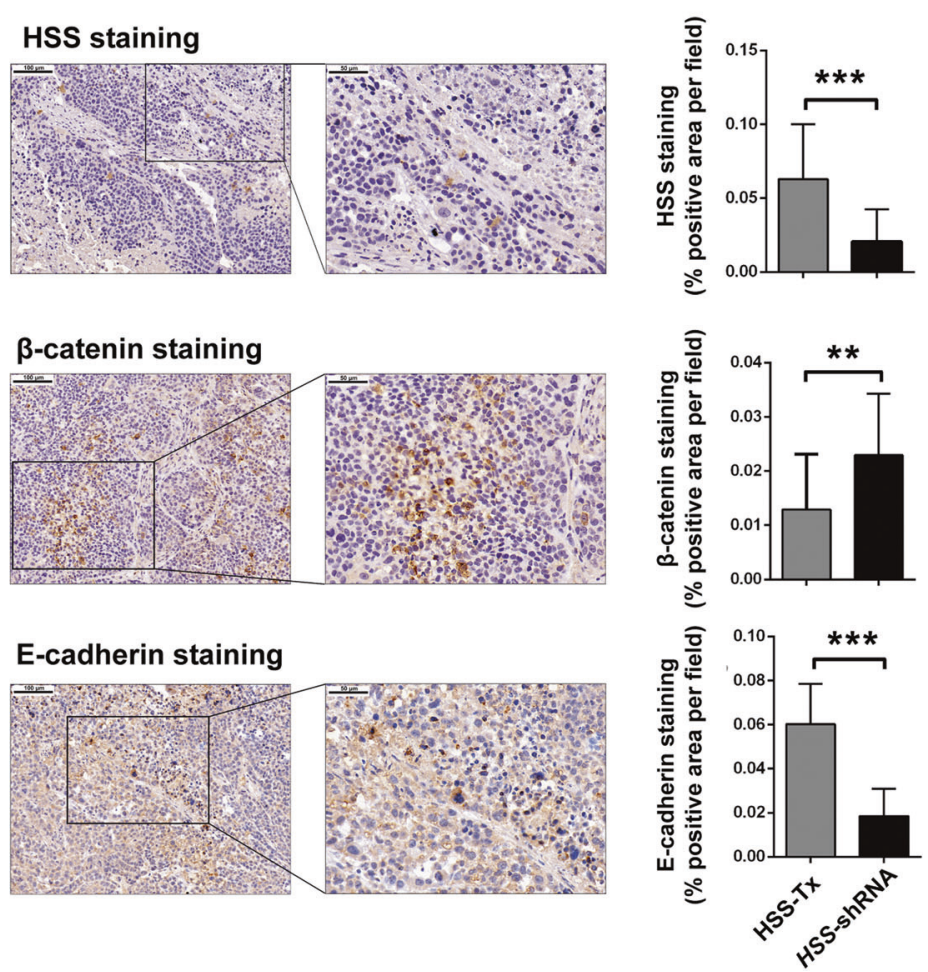

analyzed in the HSS-Tx and HSS-shRNA cells. As confirmed in Fig. 4a and b, HSS knockdown reduced the expression of epithelial markers such as E-cadherin and ZO1 and increased the expression of mesenchymal markers such as fibronectin and $\alpha$-SMA; Conversely, HSS upregulation increased the expression of E-cadherin and ZO-1 and reduced the expression of fibronectin and $\alpha$-SMA. The EMT phenomenon revealed in HCC cells was reconfirmed 
Fig. 4 EMT marker expression in HSS-Tx or HSS-shRNA HCC cells. a, $\mathbf{b}$ The expression of EMT markers in HSS-Tx and HSS-shRNA cells (HepG2 cells). Western blot analysis was performed using antibodies against the epithelial markers, E-cadherin and ZO-1, and the mesenchymal markers, $\alpha$-SMA and Fibronectin, respectively. The bands were analyzed with Image $\mathbf{J}$ software and the relative densities of the bands were normalized to GAPDH. The values are expressed as the means \pm SD of three independent experiments. c, $\mathbf{d}$ The IHC assay was conducted to detect the expressions of HSS, $\beta$-catenin, and Ecadherin in the tumors of MHCC97H cells. Tissue sections are representative of at least five samples in each group. For each section, at least six fields were randomly chosen for histological analysis. Quantitation of positive area was analyzed with Image $\mathbf{J}$ software. $* P<0.05, * * P<0.01$, and $* * * P<0.001$.

in xenograft tumors caused by MHCC97H (shown in Fig. $4 \mathrm{c}$ and $\mathrm{d}$ ), and the EMT markers in the HCC tissues exhibited similar changes to those observed in HepG2 cells. All these figures indicate that the HSS downregulation helps $H S S$-shRNA cells to maintain the mesenchymal status and results in cell migration via EMT; whereas, the HSS upregulation in HSS-Tx cells assists the HCC cells to retain in epithelial status. In short, HSS seems to be an important regulator for cell migration during EMT.

\section{HSS downregulation enhanced HCC invasion via the ERK signaling pathway}

Much solid evidence has supported the relevance of EMT for HCC progression. Snail is a family of transcription factors that significantly represses E-cadherin expression and triggers EMT efficiently [38]. Furthermore, Liu et al. [39] reported that Snail expression was positively correlated with tumor metastasis. Hence, to find out whether HSS regulated the Snail molecule and to understand its involvement in HCC metastasis was our next goal. As shown in Fig. 5a, metastatic HCC tissues with VI exhibited a profound increase both in Snail expression and in ERK phosphorylation. In contrast, in the HCC tissues without VI, Snail expression and ERK phosphorylation remained at relatively low levels. Further, we investigated whether $H S S$ shRNA could activate ERK phosphorylation, thereby triggering Snail expression. As shown in Fig. 5b, parallel to ERK phosphorylation in MHCC97H-HSS-shRNA cells, Snail expression was dominantly increased (by approximately threefold above MHCC97H-HSS-Tx cells). Figure $5 \mathrm{c}$ and $\mathrm{d}$ further demonstrated that the increased migration of HepG2 cells caused by HSS-shRNA could be efficiently suppressed by PD98059, a specific ERK inhibitor. PD98059 inhibition of ERK phosphorylation was inversely correlated with migration of HCC cells (Fig. 5e) and Snail expression (Fig. 5f-h). Taken together, these results provide a novel potential mechanism of HCC metastasis in that lack of HSS expression promoted ERK phosphorylation by an unknown modulator, triggering Snail expression and resulting in EMT progression, which favors HCC metastasis.

\section{Discussion}

HCC is the fifth most commonly diagnosed, and the third most deadly cancer worldwide [40]. Over the last three decades, HCC is the only cancer to have shown increasing mortality in the USA [41]. As in other cancer types, metastasis is not only a complex process but also the major cause of HCC-related deaths [42]. Thus, understanding the molecular mechanisms underlying the invasive and metastatic properties of $\mathrm{HCC}$ is one of the most pressing issues in HCC research. However, the detailed mechanism by which cells acquire metastatic properties remains obscure.

In this study, we found that the expression of HSS was significantly reduced in HCC tissues with vascular invasion, suggesting that HSS could be a restricting factor preventing HCC from metastasis. This finding is similar to results reported by Dayoub et al. [32], who observed that there was decreased expression of HSS in HCC samples with accompanying angioinvasion and therefore believed that HSS might have an important role as an anti-metastatic factor in HCC. In their study, the short form of HSS (15 $\mathrm{kDa}$ ) was used. It is widely accepted that $15-\mathrm{kDa}$ HSS is a sort of secretory cytokine [14], which is released from hepatocytes and binds to the hepatocyte membrane to act as a paracrine molecule through a so far unidentified receptor [43]. In our study, the longer form of HSS $(23 \mathrm{kDa})$ was used because we considered that this $23-\mathrm{kDa}$ HSS, due to its localization in the MIMS, could exert multiple functions in regulation of hepatic proliferation, differentiation, metabolism, and apoptosis. The results obtained from this study provide solid evidence of the link between HSS and HCC metastasis and the expression level of HSS in tumor tissue could be used as a prognostic index for the long-term outcome of HCC patients (Fig. 1). EMT associated with tumorigenesis may increase the motility and invasiveness of HCC cells, and malignant transformation may involve activation of signaling pathways promoting EMT [44, 45]. Accordingly, we hypothesized that the lack of HSS gene function induced EMT conversion and accelerated HCC metastasis. The results shown in Figs. 2 and 3 confirmed our hypothesis since $H S S$-shRNA cells acquired remarkable mesenchymal features and exhibited increasing migratory capacity. Protein kinase B (AKT) promotes cell cycle progression, cell survival, and tumor cell invasion. It has become evident that EMT is one of the many cellular processes subject to AKT regulation. Initially, we began to investigate whether the AKT-NF- $\kappa \mathrm{B}-$ Snail-E-cadherin axis could be included in the $H S S$-regulated EMT pathway. However, we were not able to verify the involvement of 
A

Patient Cases

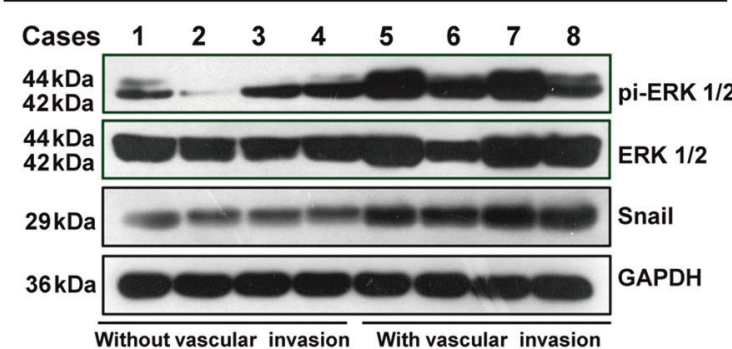

B

MHCC97H cells
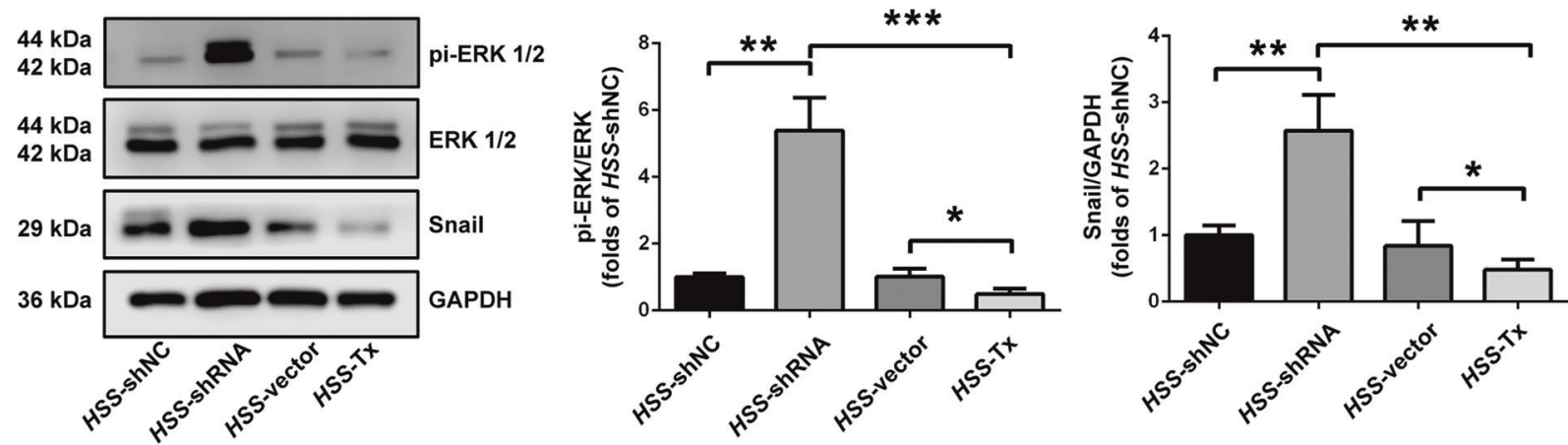

HepG2-HSS-shRNA cells
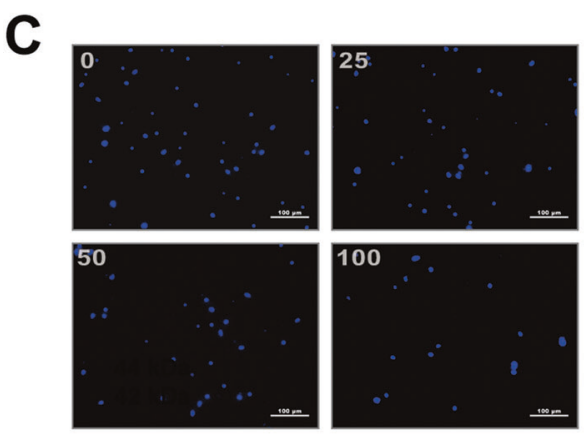

F

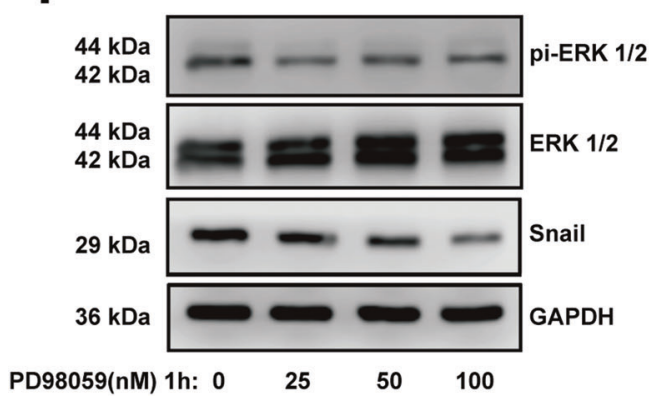

D

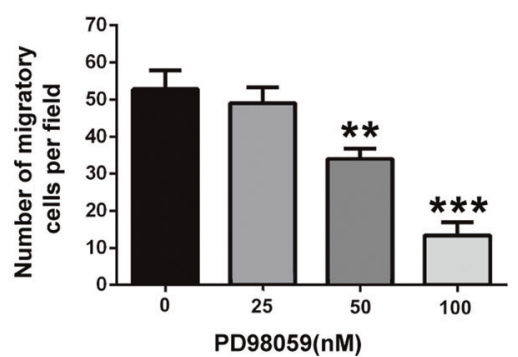

G

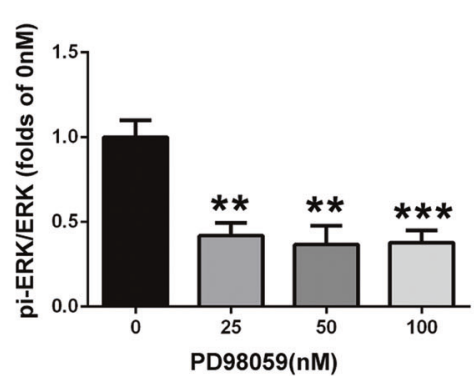

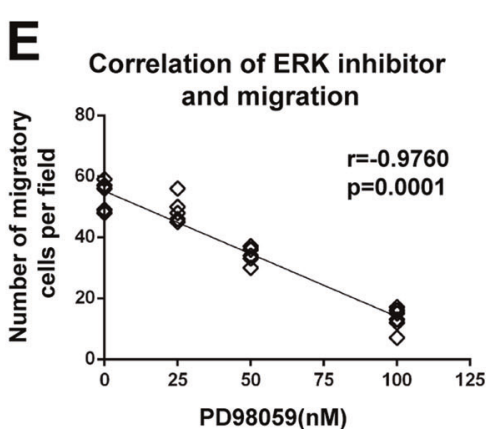

H

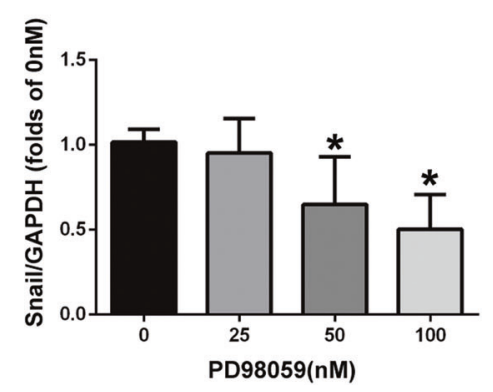

AKT and NF-кB molecules in the pathway (data not shown). Consequently, we are still seeking the potential molecule that triggers EMT in HSS-shRNA cells.
ERK signaling promotes cell invasion and motility by upregulating the Rho/Rac-actin pathway, matrix metalloproteinase (MMP) expression, and EMT-related gene 
Fig. 5 The signaling pathways of HSS that regulate EMT. a The expressions of phosphorylated ERK and Snail in HCC patient tissues with or without vascular invasion. $\mathbf{b}$ The expression of phosphorylated ERK and Snail in MHCC97H-HSS-Tx or MHCC97H-HSS-shRNA cells. The values are expressed as the means \pm SD of three independent experiments. c, d Inhibition of ERK activation by PD98059 restricted migration in HepG2-HSS-shRNA cells and exhibited a dose-effect pattern. e Pearson $r$ was equal to -0.9639 , whereas the $P$ value was 0.0001. f-h PD98059 inhibited ERK phosphorylation in HepG2-HSSshRNA cells in a dose-dependent way. HepG2-HSS-shRNA cells were treated with the different concentrations of PD98059 for $1 \mathrm{~h}$. The bands were analyzed with Image $\mathbf{J}$ software. The values are expressed as the means $\pm \mathrm{SD}$ of three independent experiments. With the increase in PD98059 dosage, the expression of pi-ERK was reduced and the snail expression was also decreased. $* P<0.05$, $* * P<0.01$, and $* * * P<0.001$.

expression; these EMT-related genes include mesenchymal genes and transcription repressors of epithelial genes, such as Snail [46, 47]. Our previous study confirmed that the downregulation of HSS expression inhibited E-cadherin expression in the early phase of liver regeneration [48], facilitating the outgrowth of parenchymal liver cells to recover the lost liver volume after PH. Following this clue, we investigated whether HSS-shRNA promoted EMT through activation of ERK phosphorylation and its downstream transcription factor Snail. As clearly indicated in Fig. 5, metastatic HCC tissues with angioinvasion exhibited elevated phosphorylation of ERK and increased Snail expression, indicating that both are essential for EMTlinked HCC metastasis. Meanwhile, we utilized MHCC97H cells, which have the highly malignant and invasive characteristics of HCC, to realize the migratory capacities regulated by $H S S$-shRNA. Similar to the results of clinical samples, MHCC97H-HSS-shRNA cells showed increased expression of phosphorylated ERK and Snail, which was also consistent with in vivo results. Similarly, HepG2-HSSshRNA cells acquired a prominent ability to migrate in the transwell assay. After a short-term incubation with the specific ERK 1/2 inhibitor-PD98059 for $1 \mathrm{~h}$, ERK phosphorylation and Snail expression were inhibited and meanwhile, migration in HepG2-HSS-shRNA cells was also restricted in a dose-dependent way. All these results confirm our hypothesis that lack of HSS contributes to HCC metastasis and that insufficient expression of HSS is, without a doubt, a deleterious factor for prognosis of patients with HCC.

In summary, expression of the 23-kDa HSS isoform has an important role in controlling HCC metastasis via ERK/ Snail-related EMT, and its expression levels could serve as a valuable prognostic index of HCC metastasis.

Acknowledgements This work was supported by the National Natural Science Foundation of China [No. 31371169].

\section{Compliance with ethical standards}

Conflict of interest The authors declare that they have no conflict of interest.

\section{References}

1. He J, Gu D, Wu X, Reynolds K, et al. Major causes of death among men and women in China. $\mathrm{N}$ Engl $\mathrm{J}$ Med. 2005;353:1124-34.

2. Farazi PA, DePinho RA. Hepatocellular carcinoma pathogenesis: from genes to environment. Nat Rev Cancer. 2006;6:674-87.

3. Kaartinen V, Voncken JW, Shuler C, et al. Abnormal lung development and cleft palate in mice lacking TGF-beta 3 indicates defects of epithelial-mesenchymal interaction. Nat Genet. 1995;11:415-21.

4. Khoury H, Dankort DL, Sadekova S, et al. Distinct tyrosine autophosphorylation sites mediate induction of epithelial-mesenchymal-like transition by an activated ErbB-2/ Neu receptor. Oncogene. 2001;20:788-99.

5. LaBrecque DR. Hepatic stimulator substance. Discovery, characteristics and mechanism of action. Dig Dis Sci. 1991;36:669-73.

6. LaBrecque DR. In vitro stimulation of cell growth by hepatic stimulator substance. Am J Physiol. 1982;242:G289-95.

7. LaBrecque DR, Steele G, Fogerty S, et al. Purification and physical-chemical characterization of hepatic stimulator substance. Hepatology. 1987;7:100-6.

8. Fleig WE, Hoss G. Partial purification of rat hepatic stimulator substance and characterization of its action on hepatoma cells and normal hepatocytes. Hepatology. 1989;9:240-248.

9. Francavilla A, Hagiya M, Porter KA, et al. Augmenter of liver regeneration: its place in the universe of hepatic growth factors. Hepatology. 1994;20:747-57.

10. Hagiya M, Francavilla A, Polimeno L, et al. Cloning and sequence analysis of the rat augmenter of liver regeneration (ALR) gene: expression of biologically active recombinant ALR and demonstration of tissue distribution. Proc Natl Acad Sci USA. 1994;91:8142-6.

11. Giorda R, Hagiya M, Seki T, et al. Analysis of the structure and expression of the augmenter of liver regeneration (ALR) gene. Mol Med. 1996;2:97-108.

12. Becher D, Kricke J, Stein G, et al. A mutant for the yeast scERV1 gene displays a new defect in mitochondrial morphology and distribution. Yeast. 1999;15:1171-81.

13. Hofhaus G, Stein G, Polimeno L, et al. Highly divergent amino termini of the homologous human ALR and yeast scERV1 gene products define species specific differences in cellular localization. Eur J Cell Biol. 1999;78:349-56.

14. Gandhi CR, Kuddus R, Subbotin VM, et al. A fresh look at augmenter of liver regeneration in rats. Hepatology. 1999;29:1435-45.

15. Wu CK, Dailey TA, Dailey HA, et al. The crystal structure of augmenter of liver regeneration: A mammalian FAD-dependent sulfhydryl oxidase. Protein Sci. 2003;12:1109-18.

16. Farrell SR, Thorpe C. Augmenter of liver regeneration: a flavindependent sulfhydryl oxidase with cytochrome $\mathrm{c}$ reductase activity. Biochemistry. 2005;44:1532-41.

17. Lange H, Lisowsky T, Gerber J, et al. An essential function of the mitochondrial sulfhydryl oxidase Erv1p/ALR in the maturation of cytosolic Fe/S proteins. EMBO Rep. 2001;2:715-20.

18. Daithankar VN, Farrell SR, Thorpe C. Augmenter of liver regeneration: substrate specificity of a flavin-dependent 
oxidoreductase from the mitochondrial intermembrane space. Biochemistry. 2009;48:4828-37.

19. Gandhi CR. Augmenter of liver regeneration. Fibrogenes Tissue Repair. 2012;9:5.

20. Polimeno L, Pesetti B, Annoscia E, et al. Alrp, a survival factor that controls the apoptotic process of regenerating liver after partial hepatectomy in rats. Free Radic Res. 2011;45:534-49.

21. Thirunavukkarasu C, Wang LF, Harvey SA, et al. Augmenter of liver regeneration: an important intracellular survival factor for hepatocytes. J Hepatol. 2008;48:578-88.

22. Francavilla A, Pesetti B, Barone $M$, et al. Transient GFER knockdown in vivo impairs liver regeneration after partial hepatectomy. Int J Biochem Cell Biol. 2014;53:343-51.

23. Han LH, Dong LY, Yu H, et al. Deceleration of liver regeneration by knockdown of augmenter of liver regeneration gene is associated with impairment of mitochondrial DNA synthesis in mice. Am J Physiol Gastrointest Liver Physiol. 2015;309:G112-22.

24. Polimeno L, Rossi R, Mastrodonato M, et al. Augmenter of liver regeneration, a protective factor against ROS-induced oxidative damage in muscle tissue of mitochondrial myopathy affected patients. Int J Biochem Cell Biol. 2013;45:2410-9.

25. Li S, Tang $\mathrm{Z}, \mathrm{Yu} \mathrm{H}$, et al. Administration of naked plasmid encoding hepatic stimulator substance by hydrodynamic tail vein injection protects mice from hepatic failure by suppressing the mitochondrial permeability transition. J Pharmacol Exp Ther. 2011;338:750-7.

26. Zhang J, Li Y, Jiang S, Yu H, et al. Enhanced endoplasmic reticulum SERCA activity by overexpression of hepatic stimulator substance gene prevents hepatic cells from ER stress-induced apoptosis. Am J Physiol Cell Physiol. 2014;306:C279-90.

27. Ilowski M, Kleespies A, de Toni EN, et al. Augmenter of liver regeneration (ALR) protects human hepatocytes against apoptosis. Biochem Biophys Res Commun. 2011;404:148-52.

28. Gandhi CR, Chaillet JR, Nalesnik MA, et al. Liver-specific deletion of augmenter of liver regeneration accelerates development of steatohepatitis and hepatocellular carcinoma in mice. Gastroenterology. 2015;148:379-91.

29. Thasler WE, Schlott T, Thelen P, et al. Expression of augmenter of liver regeneration (ALR) in human liver cirrhosis and carcinoma. Histopathology. 2005;47:57-66.

30. Gribilas G, Zarros A, Zira A, et al. Involvement of hepatic stimulator substance in experimentally induced fibrosis and cirrhosis in the rat. Dig Dis Sci. 2009;54:2367-76.

31. Margeli AP, Papadimitriou L, Ninos S, et al. Hepatic stimulator substance administration ameliorates liver regeneration in an animal model of fulminant hepatic failure and encephalopathy. Liver Int. 2003;23:171-8.

32. Dayoub R, Wagner H, Bataille F, et al. Liver regeneration associated protein (ALR) exhibits antimetastatic potential in hepatocellular carcinoma. Mol Med. 2011;17:221-8.
33. Yu HY, Zhu MH, Xiang DR, et al. High expression of $23 \mathrm{kDa}$ protein of augmenter of liver regeneration (ALR) in human hepatocellular carcinoma. Onco Targets Ther. 2014;7:887-93.

34. Guo D, Dong LY, Wu Y, et al. Downregulation of hepatic nuclear factor 4alpha on expression of human hepatic stimulator substance via its action on the proximal promoter in HepG2 cells. Biochem J. 2008;415:111-21.

35. de Longueville F, Atienzar FA, Marcq L, et al. Use of a lowdensity microarray for studying gene expression patterns induced by hepatotoxicants on primary cultures of rat hepatocytes. Toxicol Sci. 2003;75:378-92.

36. Huber MA, Kraut N, Beug H. Molecular requirements for epithelial-mesenchymal transition during tumor progression. Curr Opin Cell Biol. 2005;17:548-58.

37. Jou J, Diehl AM. Epithelial-mesenchymal transitions and hepatocarcinogenesis. J Clin Invest. 2010;120:1031-4.

38. Barrallo-Gimeno A, Nieto MA. The Snail genes as inducers of cell movement and survival: implications in development and cancer. Development. 2005;132:3151-61.

39. Liu L, Dai Y, Chen J, et al. Maelstrom promotes hepatocellular carcinoma metastasis by inducing epithelial-mesenchymal transition by way of Akt/GSK-3b/Snail signaling. Hepatology. 2014;59:531-43.

40. Parkin DM. Global cancer statistics in the year 2000. Lancet Oncol. 2001;2:533-43.

41. Ryerson AB, Eheman CR, Altekruse SF, et al. Annual report to the nation on the status of cancer, 1975-2012, featuring the increasing incidence of liver cancer. Cancer. 2016;122:1312-37.

42. Roxburgh P, Evans TR, et al. Systemic therapy of hepatocellular carcinoma: are we making progress? Adv Ther. 2008;25: 1089-104.

43. Lu C, Li Y, Zhao Y, et al. Intracrine hepatopoietin potentiates AP1 activity through JAB1 independent of MAPK pathway. FASEB J. 2002;16:90-2.

44. Hanahan Dand Weinberg RA. Hallmarks of cancer: the next generation. Cell. 2011;144:646-74.

45. Thiery JP. Epithelial-mesenchymal transitions in tumor progression. Nat Rev Cancer. 2002;2:442-54.

46. Bigatto V, De Bacco F, Casanova $\mathrm{E}$, et al. TNF- $\alpha$ promotes invasive growth through the MET signaling pathway. Mol Oncol. 2015;9:377-88.

47. Xie G, Diehl AM. Evidence for and against epithelial-tomesenchymal transition in the liver. Am J Physiol Gastrointest Liver Physiol. 2013;305:G881-90.

48. Zhang H, Dong LY, Sun G, et al. Downregulation of hepatic stimulator substance during the early phase of liver regeneration inhibits E-cadherin expression in mice. Int $\mathrm{J}$ Biochem Cell Biol. 2014;47:38-46. 